\title{
A NEW DIAGNOSTIC FOR THE MEASUREMENT OF THE IONIC EMISSION GENERATED BY AN ELECTRON-PHOTON CONVERSION TARGET
}

\author{
A. Compant La Fontaine, A. Godefroy, D. Guilhem, P. Leclerc, J.L. Lemaire and J.Y. Salmon \\ CEA/DAM Ile-de-France, BP12-F 91680, Bruyères-le-Châtel, France
}

\begin{abstract}
A beam diagnostic made of a double sheet sensor is designed to the search of ions, emitted by an electronphoton conversion target under the impact of an intense relativistic electron beam. This device is tested on the PIVAIR prototype accelerator. Signals attributed to ions emitted from the target are observed for different target elements : Ta, C, Ti, Au and light species are identified by their time of flight. The mean velocities of $\mathrm{C}+$ and $\mathrm{H}+$ ions are found to be respectively equal to $1.2108 \mathrm{~cm} / \mathrm{s}$ and $5108 \mathrm{~cm} / \mathrm{s}$ for $\mathrm{C}$ and Ta targets. The ionic appearance and rise time signals are closed to the expected values. Besides, the current signals are characterized by a plateau to which level corresponds to the Child-Langmuir current (25 A for $\mathrm{H}+$ ions). Furthermore, the fall-off of this signal is found to be in synchronism with the electron beam one. This behaviour represents the deconfinement of the ion beam under the effect of its own space charge. These experimental results confirm the preliminary calculations obtained with the M2V Maxwell-Vlasov PIC code which has been coupled with a ionic emission model.
\end{abstract}

\section{INTRODUCTION}

A high-intensity (3 $\mathrm{kA})$ relativistic $(7 \mathrm{MeV})$ electron beam is focused (during $85 \mathrm{~ns}$ ) on a tantalum target and a large fraction of the incident energy is converted in photons via the bremsstrahlung process. The X-ray emission, directed inside a cone centered along the electron beam axis is used for radiography purposes.

The energy deposited (during the interaction) on a 2 $\mathrm{mm}$ spot size vaporizes the target and ions are produced. A charge neutralization of the beam then occurs leading to a pinch of the beam. The ions are subsequently accelerated backstream in the axial electric field of the electron beam. Thus, the pinch displaces from the target along with the ions. It leads to an increase of the spot size on the target and would result in a degradation of the sharpness of the produced radiographic image.

The X-ray spot size broadening effect was observed during the pulse time duration on the prototype facilities ITS at Los Alamos [1] and PIVAIR at CESTA. It was assigned to the emission of both heavy ions issued from the target material [1], [2] and light ions $\mathrm{H}+$ issued from hydrogen atoms contaminating the target [3], [4]. They are produced by the vaporization of the target.
In order to identify the ions emitted and to check our theory of this ionic emission, a beam diagnostic made up of two conducting sheets properly screened is designed and tested on the PIVAIR facility.

\section{THE DOUBLE SHEET DIAGNOSTIC APPARATUS}

It consists of two thin carbon foils, $100 \mathrm{~mm}$ thick and 1 $\mathrm{mm}$ apart, located across the beam at a distance Y, upstream of the target, of about $2.5 \mathrm{~cm}$. The ions emitted by the target are stopped and collected only by the foil facing the target, quoted "foil 2" in figure 1 . This sheet \#2 is biased to $+100 \mathrm{~V}$ to prevent the secondary electrons to escape the foil under the ionic impact.

The net ionic current is obtained by subtracting the ionic current I1 of the sheet \#1 to the one I2 of the sheet \#2 (I2-I1)T (T, means with target). By this way, the fast signal observed at the beginning of the pulse can be eliminated as well as the signal corresponding to the backscattered electrons issued from the target and crossing the two sheets.

Of course, high energy secondary electrons are emitted at the back face of the two foils. Those produced by the electron beam on the sheet \#1 are collected by the sheet \#2. Thus, the currents produced by the electron beam on the two foils are not the same. It gives an offset which can be easily measured thanks to a shot, fired without target (the electrons of the beam are dumped on a graphite block further downstream). This value (I2-I1)noT (noT, means with no target) is then subtracted to the previous value (I2-I1)T. Hence, the final ionic current is given by the relation: $\mathrm{I}=(\mathrm{I} 2-\mathrm{I} 1) \mathrm{T}-(\mathrm{I} 2-\mathrm{I} 1) \mathrm{noT}$.

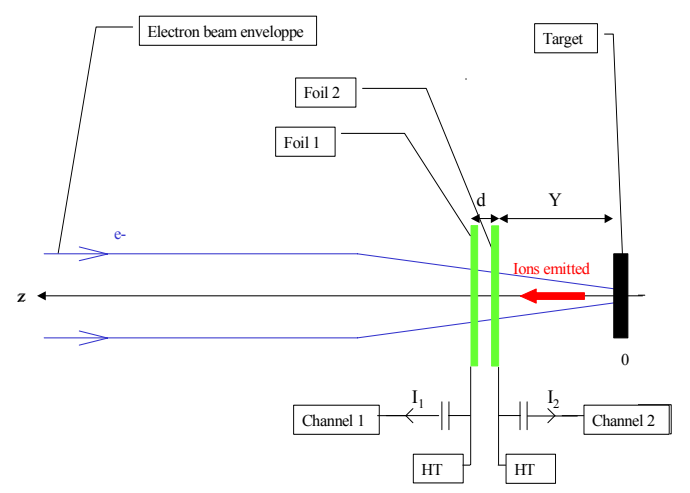

Fig. 1 : Scheme of the ionic emission measurement principle, on the PIVAIR facility 
The sheets are housed inside a Faraday cup allowing a screening effect. They are connected to a $5 \mathrm{GHz}$ scope through rigid $50 \mathrm{~W}$ coaxial cables of $150 \mathrm{MHz}$ bandwidth and of equal electric length, see figure 2 .

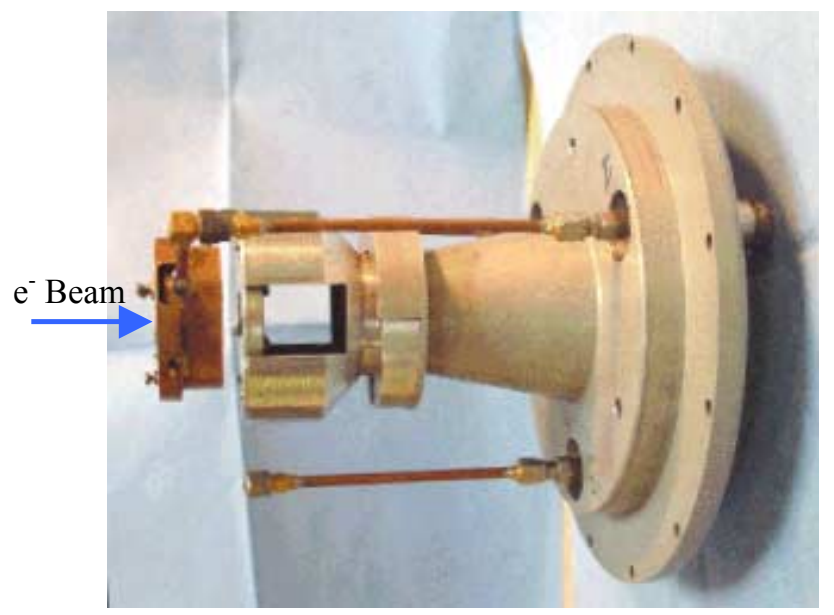

Fig. 2 : Photograph of the diagnostic apparatus

This device has been first checked on a EMC (Electro Magnetic Compatibility) testing bench. It is capable to deliver a capacitive discharge that produces an electric field $\mathrm{E} \sim 150 \mathrm{~V} / \mathrm{cm}$, during $7 \mathrm{~ns}$ (rise time of this electromagnetic perturbation). On each sheet, one observe in figure 3 a sharp signal of $0.5 \mathrm{~V}$ (followed by a few oscillations) both synchronized and with the rise time of the excitation signal.

\section{EXPERIMENTAL RESULTS}

In real experimental conditions, signals are recorded on channels 1 and 2; they are displayed in figure 4 along with the electron beam pulse current. It corresponds to a shot with a tantalum target. A short oscillation of $1 \mathrm{kV}$ amplitude is observed during the rise time of the electron beam signal. Using the EMC previous results, this behaviour is interpreted as the induction on the foils of the electromagnetic wave induced by the electron beam.

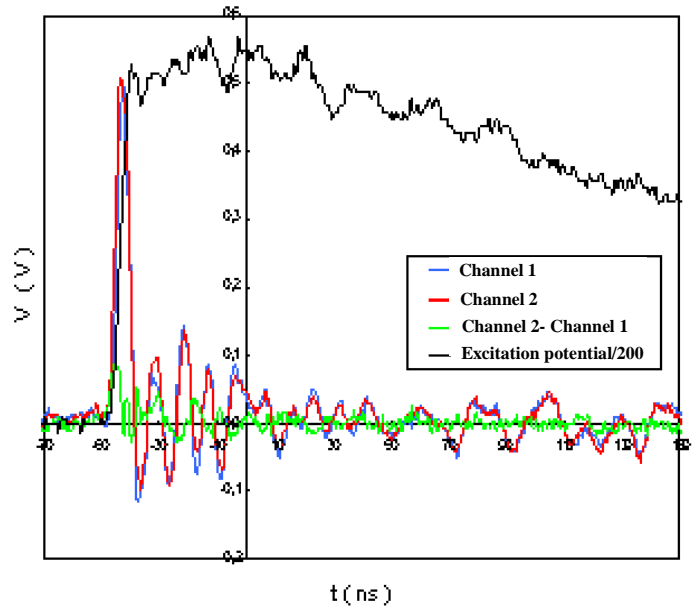

Fig. 3 : Test of the time-of-flight apparatus (testing bench delivers an electric field $\mathrm{E}=15 \mathrm{kV} / \mathrm{m}$ )
In figure 5 are shown the same signals without target. The plateaux of the currents viewed on channels 1 and 2 correspond to the emission of high energy secondary electrons produced by sheets $\# 1$ and \#2, when the primary electron beam crosses the foils.

The differences (I2-I1)T and (I2-I1)noT shown in figure 6 and obtained respectively from figures 4 and 5 leads to the searched ionic current I= (I2-I1)T - (I2I1)noT.

By using a carbon target, ionic signals are obtained for two foil-target spacing, namely $\mathrm{Y}=1 \mathrm{~cm}$ and $4.5 \mathrm{~cm}$, see figure 7 . The transit time of the $\mathrm{C}+$ ions (the only species expected to be produced) $7.3 \mathrm{~ns}$ per $\mathrm{cm}$, is deduced from these signals. The mean velocity is $\mathrm{v}(\mathrm{C}+) \exp =1.4 \times 108$ $\mathrm{cm} / \mathrm{s}$.

Similar results obtained with a Ta target are shown in figure 8. Transit time of $\mathrm{H}+$ ions is $2 \mathrm{~ns}$ per $\mathrm{cm}$, and mean velocity is $\mathrm{v}(\mathrm{H}+) \exp =5 \times 108 \mathrm{~cm} / \mathrm{s}(\mathrm{H}+$ ions are identified by doing the ratio of $\mathrm{H} / \mathrm{C}$ ion masses). For both cases, the ionic current is close to the Child-Langmuir theoretical current limit ( $6 \mathrm{~A}$ and $20 \mathrm{~A}$ for $\mathrm{C}+$ and $\mathrm{H}+$ ions respectively). About the same values are obtained with the Maxwell-Vlasov code M2V [4].

It can be checked in figure 9 that no $\mathrm{H}+$ ion signal is observed for a defocused beam, tuned to $1 \mathrm{~cm}$ beam radius at the target. On the same figure, the ionic emission initiated by a focused electron beam, ends with the electron beam pulse. This behaviour is interpreted as an explosion of the ion beam under the effect of its own space charge, since it is not confined anymore by the electron beam potential.

\section{CONCLUSION}

The ionic currents are obtained with a signal to noise ratio of about 15, sufficient enough to show the ionic emission of the light ions $\mathrm{C}+$ and $\mathrm{H}+$ issued from the targets. Heavy $\mathrm{Ta}+$ ions probably exist, but are not observed, because they are expected to reach the foil \#2 after the end of the beam pulse. These results confirm the ionic emission hypothesis [1-4].

Moreover, this emission is found to last until the end of the beam duration. This shows that the hydrogen atoms are present as an hydride formed in the target, as $\mathrm{TaH}$, more likely than adsorbed on a few monolayers [4]. The amount of hydrogen atoms absorbed in the tantalum target is being analysed by means of nuclear micro-probe.

\section{REFERENCES}

[1] T. Kwan, "Electron Beam-Target Interaction in the X-Ray Radiograpy", LA-UR-98-4802, LANL (1996).

[2] C. Vermare, J. Labrouche, P. Le Taillandier de Gabory, D. Villate and J. T. Donohue, "Strong SelfFocusing of a $7.2 \mathrm{MeV}$ Electron Beam Striking an Aluminized Mylar Target”, IEEE Transaction on Plasma Science, Vol.27, №6 (1999) 1566.

[3] C. Vermare, J. Labrouche, P. Le Taillandier de Gabory, D. Villate and J. T. Donohue, "Observation of Strong Self-focusing of an Intense Relativistic Electron 
Beam Impinging on a $\mathrm{SiO} 2$ Target", IEEE Transaction on Plasma Science, Vol.27, №5 (1999) 1501.

[4] A. Compant La Fontaine, J. L. Lemaire, C. Quine and J. Segré, "Numerical Simulations and Experimental Aspects of Space Charge Compensation in a High Energy Electron Beam", Proceedings of EPAC 2000, Vienna, Austria (2000) 1309.

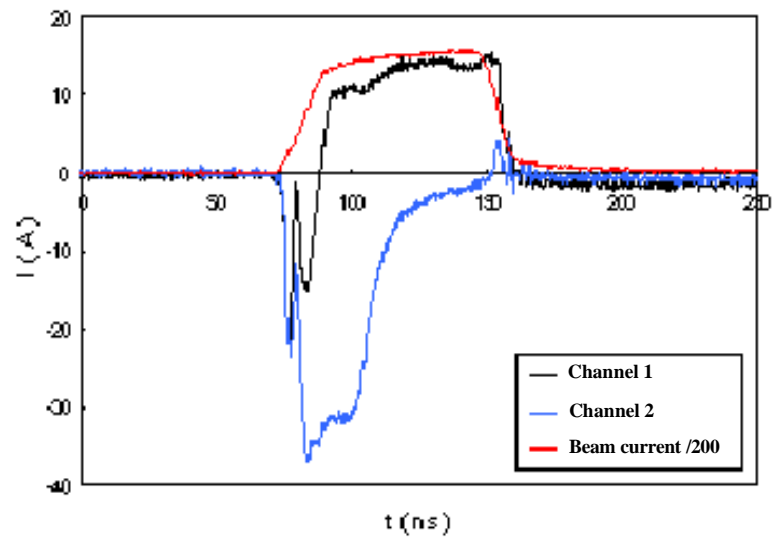

Fig. 4 : IT1 and IT2 currents on foils, shot with tantalum target; Ibeam $=3 \mathrm{kA}, \mathrm{Y}=2.5 \mathrm{~cm}$

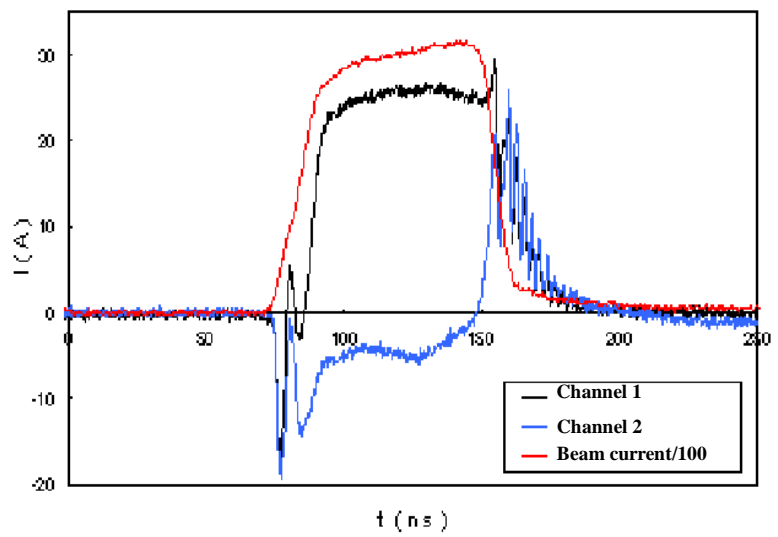

Fig. 5 : InoT1 and InoT2 currents on foils, shot without target; Ibeam $=3 \mathrm{kA}, \mathrm{Y}=2.5 \mathrm{~cm}$

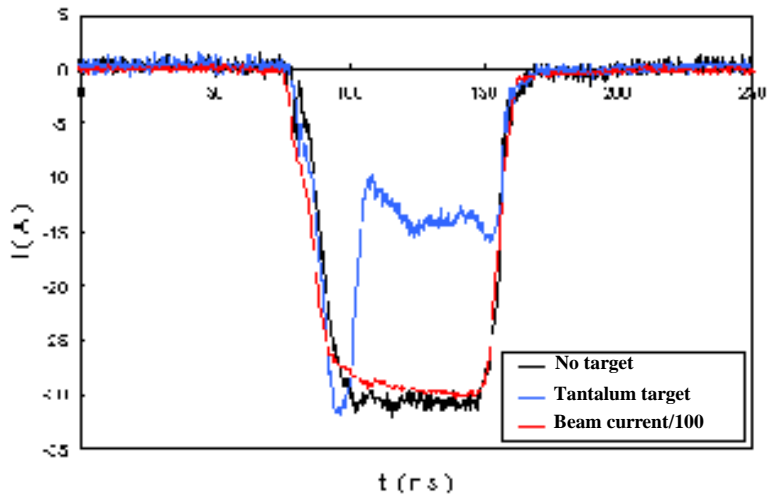

Fig. 6 : (I2-I1)T and (I2-I1)noT relative foil currents, respectively with Ta target and without target; Ibeam $=3$ $\mathrm{kA}, \mathrm{Y}=2.5 \mathrm{~cm}$

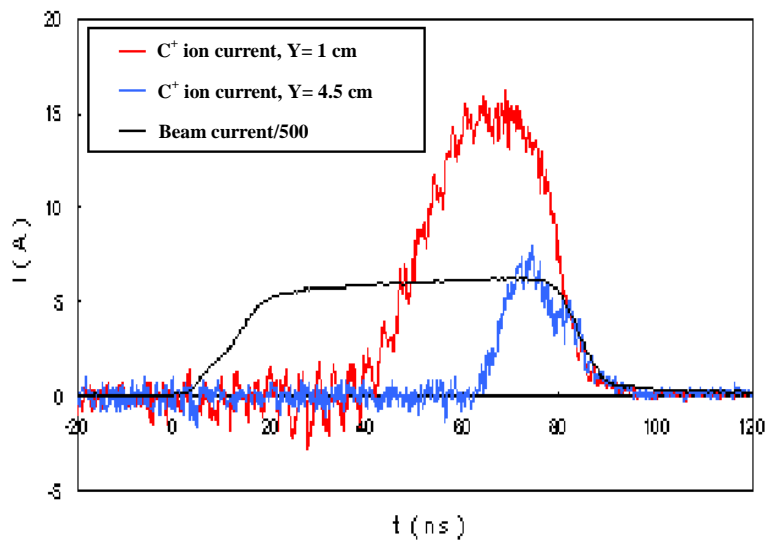

Fig. 7 : Time of flight of $\mathrm{C}+$ ions, carbon target, ionic current measured : Iions $=($ I2-I1)T-(I2-I1)noT; Ibeam $=3$

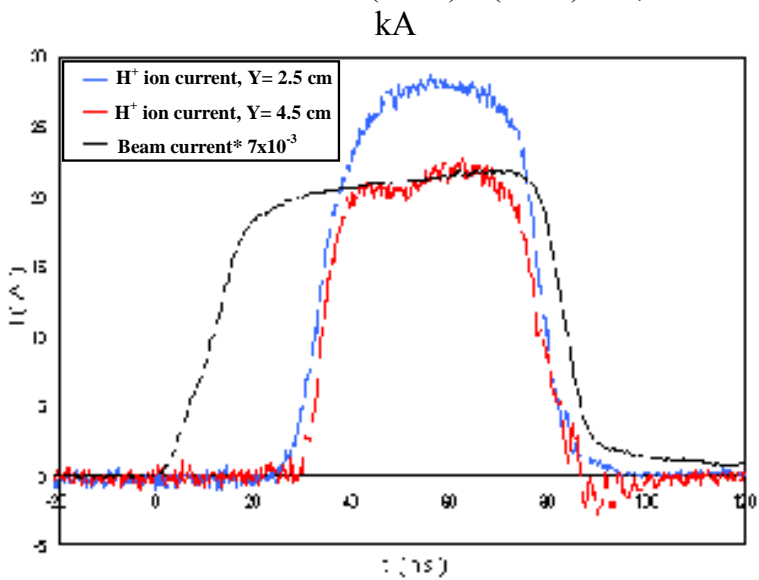

Fig. 8 : Time of flight of $\mathrm{H}+$ ions, tantalum target, ionic current measured : Iions $=($ I2-I1)T-(I2-I1)noT; Ibeam $=3$

$\mathrm{kA}$

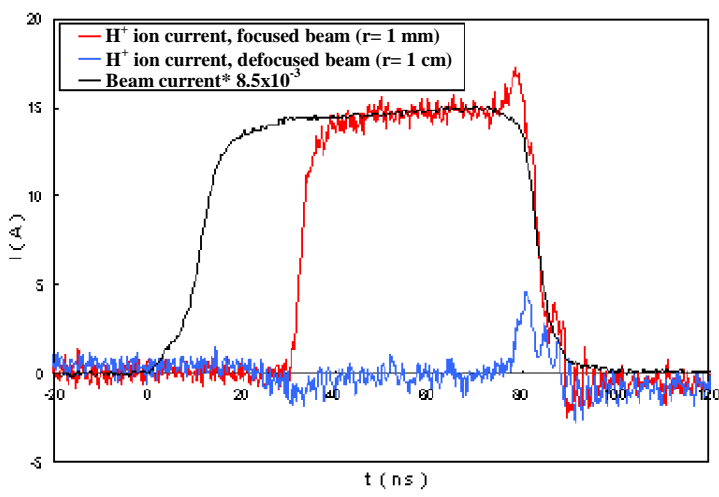

Fig. 9 :Effect of strong defocusing of the electron beam on Ta target, ionic current measured : Iions $=$ (I2-I1)T-(I2I1)noT; Ibeam=1.7 kA, Y=2.5 cm 